\title{
H1N1 Influenza
}

National Cancer Institute

\section{Source}

National Cancer Institute. H1N1 Influenza. NCI Thesaurus. Code C80444.

An acute viral respiratory infection caused by a strain of influenza virus which is endemic in swine (pigs). Rarely reported in humans prior to 2009, the disease is caused by a mutated strain of swine influenza A (H1N1) virus. It is highly contagious and spreads mainly through coughing and sneezing. Signs and symptoms include fever, chills, coughing, sore throat headache, muscle ache, and generalized weakness. Antiviral medications are most effective in the first two days of the illness. 